\title{
PREDICTABILITY OF FUTURE INDEX RETURNS BASED ON \\ THE 52-WEEK HIGH STRATEGY
}

\author{
Mirela Malin*\# and Graham Bornholt*
}

\begin{abstract}
In a landmark paper, George and Hwang (2004) show that a stock's 52-week high price largely explains the mom entum effect and that a strategy based on closeness to the 52 week high has better forecasting power for future returns than do momentum strategies. We find that the 52-week high strategy is unprofitable when applied to emerging markets indices, and that it is significantly less profitable than the corresponding momentum strategy. Overall the 52-week high effect is not as pervasive as the momentum effect.
\end{abstract}

Key words: 52-week high; momentum; emerging markets; index returns;

JEL classification: G14, G15

\footnotetext{
*Griffith Business School, Griffith University, Gold Coast, Australia

\# Corresponding Author: Dr Mirela Malin, Griffith Business School, Department of Accounting Finance and Economics, Gold Coast Campus, Griffith University, Queensland, 4222, Australia; Email: m.malin@griffith.edu.au; Telephone: +61 075552 7719; Fax: +61 0755528068.

We thank Jerry Bowman, Stefano Cavaglia, and an anonymous referee for helpful comments.
} 


\section{Introduction}

The empirical literature on momentum strategies has demonstrated that past returns of stocks can be used to predict future returns ${ }^{1}$. Studies that have investigated the momentum effect have confirmed the persistence of this anomaly at company, industry and international index level ${ }^{2}$. Various explanations for momentum have been proposed in the literature but its cause remains controversial. Behavioral models have been proposed to explain return predictability as a consequence of investor irrationality induced by psychological biases. Barberis, Shleifer and Vishny (1998) (BSV hereafter) explain momentum as an underreaction to information as the result of conservatism bias. While contrary to BSV, Daniel, Hirshleifer and Subrahmanyam (1998) (DHS hereafter) suggest that overreaction is the source of momentum. A third model, proposed by Hong and Stein (1999) (HS hereafter), explains momentum as a result of slow and gradual dissemination of information.

Related research has investigated whether other variables that are associated with returns can have predictive power. Daniel and Titman (1999) find that stocks with low book-to-market ratio exhibit stronger return continuation, evidence consistent with DHS model of overreaction. Chan, Jegadeesh and Lakonishok (1999) show that both price and earnings momentum yield significant returns resulting from underreaction to information, in line with BSV model. Lee and Swaminathan (2000) show that past trading volume is useful in predicting price momentum, a result that is consistent with the gradual diffusion of information in the HS model.

George and Hwang (2004) use a stock's price levels, in the form of the 52-week high (52wk hereafter), to show that closeness to the 52wk high explains a large part of stock price momentum. Their measure of closeness to the 52wk high is the ratio of current price divided by the $52 w k$ high price of a share. The $52 w k$ high strategy proposed goes long in the portfolio

\footnotetext{
${ }^{1}$ Jegadeesh and Titman (1993), (2001), Fama and French (1996), Moskowitz and Grinblatt (1999), Grundy and Martin (2001), Lewellen (2002), Chan, Hameed and Tong (2000).

${ }^{2}$ Rouwenhorst (1998), Griffin, Ji and Martin (2003), Balvers and Wu (2006), Bhojraj and Swaminathan (2006).
} 
of stocks that have high 52wk high ratios and shorts the portfolio of stocks with low $52 \mathrm{wk}$ high ratios. Their analysis compares this strategy with a pure momentum strategy based on Jegadeesh and Titman (1993) and with the industry momentum strategy of Moskowitz and Grinblatt (1999). Their results reveal that price levels are better determinants of return continuation effects than past price changes, and that the $52 \mathrm{wk}$ high measure has predictive power whether or not stocks have experienced extreme returns in the past.

George and Hwang (2004) claim that the 52wk high strategy dominates the corresponding momentum strategy. Their explanation for return continuation is different to the explanations given by the existing behavioral models. They believe that return continuation based on the $52 \mathrm{wk}$ high ratio is consistent with the "adjustment and anchoring bias" whereby traders use the 52wk high as an anchor when adjusting stock values as new information arrives. In a similar fashion to earlier momentum studies, George and Hwang (2004) test whether the 52wk high strategy profits eventually reverse. Their results indicate no evidence of reversals, suggesting that the $52 \mathrm{wk}$ high effect is a distinct phenomenon from short-term continuation, and that this effect might be triggered by a different behavioral bias. Motivated by the George and Hwang study, Marshall and Cahan (2005) investigate whether the 52wk high persists in a different sample of stocks. Analyzing all stocks listed on the Australian Stock Exchange (ASX) that were approved for short-selling for the period January 1990 to December 2003, their study finds that the 52wk high strategy is very profitable on both small and large stocks, and on both liquid and illiquid stocks. Furthermore, the strategy achieves better results after risk adjustment and outperforms both the original pure momentum strategy of Jegadeesh and Titman (1993) and the industry momentum strategy of Moskowitz and Grinblatt (1999) when applied to Australian stocks.

More recently, Du (2008) tests for a 52wk high effect in international market indices using a sample of 18 developed countries that covers the period from December 1969 to July 2004. Replicating the methodology of George and Hwang (2004), he shows that price levels dominate past returns in terms of predictive power when the 52wk high strategy is paired with the momentum strategy. This is consistent with the original findings of George and 
Hwang (2004) that are based on individual stocks. However, when comparing the two individual strategies, Du's results indicate that momentum profits exceed the 52wk high strategy's profits, even after risk adjustment. Furthermore, Du (2008) provides evidence that continuation of returns coexists with the eventual reversal of returns for both the momentum and 52wk high strategy. This result contradicts George and Hwang's (2004) findings. Although Du (2008) agrees with the anchor and bias explanation of the 52wk high effect, his study suggests that an overreaction can still occur when investors correct their initial bias.

The research in this paper is motivated by the need to test whether the $52 \mathrm{wk}$ high effect is as pervasive as the widely observed momentum effect. Du (2008) reports evidence of a $52 w k$ high effect in a study of developed market indices. Given that emerging markets are widely considered to be less efficient than developed markets, our expectation is that the 52wk high strategy will be more profitable when applied to emerging markets indices. Thus, our study tests for the existence of a 52wk high effect in emerging markets indices.

In contrast to the results for developed markets indices, the 52wk high strategy applied to emerging markets indices yields negative and insignificant returns. Moreover, the corresponding momentum strategy is significantly more profitable than the $52 \mathrm{wk}$ high strategy. Overall, this suggests that the $52 \mathrm{wk}$ high effect is not as pervasive as the momentum effect has proven to be.

The problem with the $52 \mathrm{wk}$ high strategy is that its short portfolio earns abnormally large future returns. We propose a possible explanation for this phenomenon based on volatility. The short portfolio may be unintentionally selecting higher volatility indices since it is composed of indices with price levels far below their 52wk highs, and higher volatility indices tend to have higher average returns. Tests support this explanation. When we modify the short portfolio by including only indices with relatively low recent past volatility, this modified short portfolio does not have abnormally large future returns.

The remainder of the paper is organized as follows: Section 2 presents the data and the empirical methodology employed in this paper. Section 3 presents the empirical results 
and robustness checks. Section 4 concludes the paper with a summary of the findings and discussion.

\section{Data and Methodology}

We use monthly prices with reinvested gross dividends and the 52wk high price of 26 Morgan Stanley Capital International (MSCl) emerging markets indices for our data set. The nearness to the $52 \mathrm{wk}$ high for each index is derived from these monthly prices. Consistent with other research at index level such as Balvers and Wu (2006) and Du (2008), the data has been downloaded from Datastream in US dollars. Returns are measured in US dollar to facilitate the interpretation of results across markets, and because the various strategy profits reflect the results that would be available to a US dollar-based investor. The time frame for the study extends from January 1988 to March 2009 with the number of observations for an index varying from 255 to 171.

Table 1 provides descriptive statistics for the distributions of returns for each of the countries included in the sample. As expected, there is a large variation in the mean and standard deviation of returns. Argentina, Brazil, Russia and Turkey have the largest monthly averages (over $2 \%$ per month), while China has the lowest average at $0.27 \%$. The 26 emerging markets countries have an average monthly return of $1.35 \%$ and an average standard deviation of $10.82 \%$. Both these values are large compared to the corresponding values for developed markets ${ }^{3}$. In terms of the distribution of returns, there is a wide range of skewness and kurtosis values, with Poland a stand-out case.

\footnotetext{
${ }^{3}$ For example, the average monthly return and standard deviation for the $18 \mathrm{MSCl}$ developed markets indices are $1.03 \%$ and $6.61 \%$, respectively.
} 
The purpose of this paper is to determine whether the $52 \mathrm{wk}$ high effect is present at index level for emerging markets, and to compare the profitability of the $52 \mathrm{wk}$ high strategies with the corresponding momentum strategies. A description of these two strategies is presented next.

\subsection{The 52wk High Strategy}

The 52wk high strategy is based on George and Huang (2004) as follows: each month $t$, indices are ranked based on the magnitude of each index i's ratio of its price at the end of month $t-1$ to the highest price achieved by index $i$ in the 12 months prior to $t-1$ :

$$
\text { Ratio }=\frac{P_{i, t-1}}{52 w k h i g h_{i, t-1}}
$$

This ratio is calculated for each index from the raw monthly closing price and the raw monthly 52wk high price. Based on the ratio ranking, every month the High $(\mathrm{H})$ portfolio contains that $25 \%$ of indices with the highest $52 w k$ high ratios, and the Low (L) portfolio consists of that $25 \%$ of indices with the lowest $52 \mathrm{wk}$ high ratios. If the 52wk high effect applies at the international index level, then indices with prices near to their 52wk high will outperform those indices that are farther from their 52wk high. Therefore the 52wk high trading strategy buys the High portfolio and sells the Low portfolio to form the High minus Low (denoted $\mathrm{H}-\mathrm{L}$ ) arbitrage portfolio. We test this for holding periods of $K=1,3,6,9$ and 12 months.

The 52wk high strategy uses overlapping portfolios with $K$ month holding periods according to the Jegadeesh and Titman (1993) methodology. This means that each month $t$ the strategy's High (Low) portfolio is comprised of $1 / K$ of the High (Low) portfolio selected in the current month as well as in the past $K-1$ months. The strategy's portfolios are revised every month $t$ as the positions initiated in $t-K$ are closed. Following George and Hwang (2004), we allow a one month gap between the end of the ranking period and the beginning of the holding period. To facilitate comparison between the 52wk high strategy and the 
momentum strategy, the holding period of $K=6$ months will serve as the base case to be discussed in detail.

\subsection{Momentum Strategy}

At the beginning of each month short term winner and short term loser portfolios are formed (each containing $25 \%$ of the indices), based on the past $J$-month returns, for $J=3,6$, 9 and 12 months. Each month $t$, the strategy buys the short term winner (SW) portfolio consisting of the $25 \%$ of indices that have the highest past returns, and sells the short term loser (SL) portfolio comprised of the $25 \%$ of indices that have the lowest past returns. According to the momentum effect, short term winners over the past three to twelve months should continue to outperform short term losers over the following three to twelve months. Therefore, the momentum strategy buys the SW portfolio and sells the SL portfolio to form the SW-SL momentum arbitrage portfolio. The SW-SL arbitrage portfolio is held for a $K$ month holding period for $K=1,3,6,9$ and12 months. To conserve space, only the strategy based on $J=6$ months will be shown, while the $K=6$ case will serve as the base case for detailed discussion.

As with the 52wk high strategy, the momentum strategy employs the overlapping portfolio approach of Jegadeesh and Titman (1993), meaning that each month's strategy return is the average of the individual portfolio returns from previous months. For example, if we take the base case strategy where $K=6$ months, the SW-SL arbitrage portfolio for a particular month contributes one-sixth of the strategy's return in each of the six following months. This approach has the advantage of reducing transaction costs and it avoids overlapping returns.

\section{Analysis of Results}

Subsection 3.1 presents the results of the 52wk high and momentum strategies for emerging countries. Subsection 3.2 provides the robustness checks of the $52 \mathrm{wk}$ high 
strategy where we present the subperiod analysis while in subsection 3.3 we show the riskadjustment regressions.

\subsection{The 52wk High and Momentum Strategies}

The results of the 52wk high strategies are presented in Table 2. It reports the average monthly US dollar returns of the Low, High and arbitrage portfolios of the $52 \mathrm{wk}$ high strategy applied to the MSCl emerging markets indices. Since the 52wk high ratio is the basis of this strategy, the formation period is 12 months.

$<$ Table 2 about here $>$

The figures indicate that the strategy is not successful. The $K=6$ base case $H-L$ loses $0.27 \%$ per month, although this figure is not significantly different from zero. More generally, the results in Table 2 show that in all cases the $H$ - $L$ strategy earns negative returns that are not significantly different from zero. This indicates that the $52 \mathrm{wk}$ high strategy does not work in emerging markets. The problem seems to be that the short portfolio $L$ is too profitable. For example, portfolio $L$ has a significant average return of $1.74 \%$ per month for the $K=6$ case $(t$-value 2.88$)$.

In contrast, the momentum strategy results reported in Table 3 indicate that the momentum strategy SW-SL generates positive profits. For the $K=6$ base case the momentum strategy has positive profits of $0.84 \%$ per month although this is only weakly significant at the $10 \%$ level ( $t$-value 1.87). More importantly, the difference in profitability between the momentum and 52 wk high strategies $(0.84 \%$ versus $-0.27 \%)$ is highly significant (the paired two-sample test $t$-value is 3.14).

$<$ Table 3 about here $>$

In summary, the $52 \mathrm{wk}$ high strategy is not profitable for emerging market indices and the momentum strategy is significantly more profitable than the $52 \mathrm{wk}$ high strategy when 
$K=6$. This means that the $52 \mathrm{wk}$ high strategy does not dominate the conventional momentum strategy in terms of profitability when these strategies are applied to emerging market indices.

\subsection{Post-holding Returns of 52wk High Portfolios}

Perhaps the behavior of the 52wk high strategy in the post-holding period will shed some light on why it fails for the emerging markets indices. Table 4 contains the post-holding period average returns for the $52 \mathrm{wk}$ high strategy with $K=1$ for the first four quarters and the first five years following the start of the holding period.

$<$ Table 4 about here $>$

The post-holding period evidence for the emerging markets indices shows a pattern of continuing losses for the 52wk high strategy. The long portfolio $H$ significantly underperforms the short portfolio $L$ in the fourth quarter, second year and fourth year after the start of the holding period. Figure 1 presents the cumulative post-holding period returns of the $52 w k$ high strategy with $K=1$ for the emerging indices for the first 60 months following the beginning of holding period. The graph tells a similar story to Table 4 . The losses in the emerging markets continue for the whole period.

<Figure 1 about here>

\subsection{Subperiod Analysis}

In this section we check the consistency of the 52wk high strategy over time by investigating the profitability of the strategy in two subperiods of approximately equal size. The first subperiod covers January 1988 to December 1998. The second subperiod covers January 1999 to March 2009. Table 5 presents the profitability of the $52 w k$ high strategy in these two subperiods. 
Panel A presents the first subperiod results and shows insignificantly negative returns for all $K$. As is the case with the full sample results, the $52 \mathrm{wk}$ high strategy profits in the first subperiod are all negative for emerging markets indices. The second subperiod results in Panel B tell a similar story: the 52wk high strategy makes losses in all cases.

$<$ Table 5 about here $>$

Figure 2 charts the subperiod post-holding cumulative monthly returns of the $52 \mathrm{wk}$ high strategy with $K=1$. It indicates somewhat different post-holding period behavior in the two subperiods. While both graphs decline across the 60 months after the start of the holding period, the decline is more gradual in the second subperiod.

$<$ Figure 2 about here $>$

\subsection{Risk-Adjusted 52wk High Profits}

Next we investigate the effect of risk adjustment on the performance of the 52wk high portfolios and strategy. In this paper the raw returns of the 52wk high strategy are riskadjusted using the two-factor and three-factor time-series regression models employed by Balvers and Wu (2006).

The two-factor model contains a world market factor and a value minus growth factor (VMG) as follows:

$$
R_{p, t}-R_{f, t}=a_{p}+b_{p, w l d}\left(R_{w l d, t}-R_{f, t}\right)+b_{p, v m g} V M G_{t}+e_{t}
$$

The second model used for risk adjustment is the international version of the Fama and French three-factor model:

$$
R_{p, t}-R_{f, t}=a_{p}+b_{p, w l d}\left(R_{w l d, t}-R_{f, t}\right)+b_{p, s m b} S M B_{t}+b_{p, h m l} H M L_{t}+e_{t}
$$

The dependent variable $R_{p, t}-R_{f, t}$ is the monthly excess return of an equallyweighted portfolio of interest, whether it's the High, Low or arbitrage portfolio of a strategy, 
where $R_{p, t}$ represents the monthly US dollar return of portfolio $p$ at time $t$ and $R_{f, t}$ is the monthly risk-free rate at time $t$ represented by the 1-month US T-bill return. The independent variables or factors for the two models are as follows: $R_{w l d, t}-R_{f, t}$ corresponds to the excess return on the MSCI World market portfolio $R_{\text {wld }}$ at time $t ; S M B_{t}$ and $H M L_{t}$ are the monthly Fama-French size and book-to-market factors ${ }^{4}$ at time $t ; V M G_{t}$ or Value minus Growth is the return on the MSCI World Value Index minus the return on the MSCI World Growth Index at time $t$.

The monthly values for the $\mathrm{MSCl}$ world market index as well as the world value and growth indices have been downloaded from the $\mathrm{MSCl}$ website ${ }^{5}$. The monthly return values for the Fama and French factors and the Ibbotson and Associates Inc. 1-month T-bill risk-free rate covering the full sample period from January 1970 to March 2009 have been downloaded from Kenneth French's website ${ }^{6}$. The risk-adjustment covers the full sample period January 1988 to March 2009 for both models. The (beta) coefficients $b_{p}$, wld, $b_{p \text {, smb }}$, $b_{p, h m l}$, and $b_{p, v m g}$ are the regression loadings corresponding to the factors of the models while the intercept $a_{p}$ (or simply alpha) represents the risk-adjusted abnormal returns of the portfolios over the estimation period. If alpha is significantly different from zero, then this is evidence of abnormal profits. The regression $t$-values are corrected for heteroskedasticity using the White (2000) test.

Table 6 reports the regression coefficients of the two models and the corresponding White-corrected $t$-statistics for the Low, High and arbitrage portfolios for the $K=6$ base case. The unadjusted annualized raw average return of each portfolio is displayed in column 2 of the table, while the annualized alpha of the two-factor and three-factor models is reported in columns 3 and 7 , respectively.

\footnotetext{
${ }^{4}$ Downloaded from Kenneth French website

${ }^{5}$ http://www.mscibarracom/products/indices/stindex

${ }^{6}$ http://www.mba.tuck.dartmouth.edu/pages/faculty/ken.french/data_library
} 
The regression results in the table indicate negative and insignificant arbitrage alphas for both models ( -0.017 and -0.034 for the two-factor and three-factor models, respectively). It is not surprising that risk adjustment of the arbitrage portfolio's results fails to find significant alphas given that the corresponding unadjusted $H-L$ profit result in Table 2 is insignificantly negative. What is of particular interest in Table 6 is the effect of risk adjustment on the profits of the long and short portfolios. Table 6 reveals that the returns of the individual short $(L)$ and long $(H)$ portfolios remain high and significant after their raw returns are adjusted for risk. The short portfolio has significant alphas of at least 0.144 while the long portfolio has significant alphas of at least 0.11 . This suggests that investors can derive annualized abnormal returns of at least $14.4 \%$ from the $L$ portfolio and at least $11.0 \%$ on the $H$ portfolio.

One possible explanation for both emerging markets portfolios having significantly positive alphas is that emerging markets as a group have abnormally high returns. This explanation was tested by risk-adjusting the $\mathrm{MSCl}$ emerging market value-weighted market index (results not shown in the table). Both risk-adjustment models produced insignificant alphas in this case $(0.029$ and 0.036 with corresponding $t$-values 0.77 and 0.93 , respectively). As a result, we can rule out the idea that the emerging market's High and Low portfolios only produce abnormal returns because emerging markets as a group produce abnormal returns.

The source of the problem with the 52wk high strategy is that the short portfolio $(L)$ earns abnormally high returns: it is not sensible to short a portfolio that has alpha exceeding $14 \%$. This is a feature of the emerging markets indices that is not present if the same analysis is done using developed markets indices. In the latter case we can report that the short portfolio of the 52wk high strategy based on $18 \mathrm{MSCl}$ Developed Markets indices has negative and insignificant alphas (results available on request). In the next section we 
suggest a possible reason why the short portfolio in the emerging markets case earns large returns.

\subsection{Volatility and the 52wk High Strategy}

The main distinguishing features of emerging market returns compared to developed market returns is that emerging markets tend to have larger average returns and much larger volatility. Perhaps it is these features that are driving the anomalous result. Portfolio $L$ is composed of emerging markets indices with values far below their 52wk highs. Shorting such indices assumes that these indices are likely to continue on a downward trend in the near future. However, the results in Table 6 have shown that such an assumption is incorrect.

Our alternative explanation is based on volatility. The portfolio composed of those indices furthest from their $52 \mathrm{wk}$ highs is likely to contain many of the more highly volatile indices. Extreme movements in prices tend to come from the more highly volatile indices. Thus these indices are likely to be over-represented in the portfolio $(L)$ of indices far from their $52 \mathrm{wk}$ highs. The problem for portfolio $L$ is that high volatility is usually associated with high average returns ${ }^{7}$. Instead of the indices in portfolio $L$ continuing on a downward trend perhaps the majority of its indices are the relatively higher return/higher volatility indices that are more likely to have large future returns. Perhaps this explains why the recent poor performances of the indices selected for $L$ tend then to be followed by high returns.

We test this volatility explanation by modifying the $52 \mathrm{wk}$ high strategy using a double sorting procedure. Within each of the $L$ and $H$ portfolios we further sort indices based on their past volatilities into small volatility $(S v)$, medium volatility $(M v)$ and big volatility $(B v)$, where each indices' past volatility is measured by its return standard deviation over the past 12 months. Let $L S v$ denote a portfolio composed of the $33 \%$ of indices in portfolio $L$ with the smallest return standard deviations over the past 12 months. The expectation is that $L S v$ will be a better candidate for shorting than portfolio $L$ because those indices in $L$ with relatively

\footnotetext{
${ }^{7}$ The correlation between average monthly return and standard deviation in Table 1 is +0.635 .
} 
higher recent volatility have been eliminated from LSV. We also consider whether the long portfolio can be improved by eliminating relatively low volatility indices. Therefore, let $H B V$ denote a portfolio composed of the $33 \%$ of indices in portfolio $H$ with the biggest return standard deviations over the past 12 months. The modified 52wk high long/short strategy is denoted HBV-LSv.

The raw results of the modified 52wk high strategies for $K=1,3,6,9$, and 12 months are presented in Table 7. It reports the average monthly US dollar returns of the $L S v$, $H B v$ and arbitrage portfolios $H B v-L S v$. While four of the arbitrage profits are now positive, none are significant. In comparison to the standard 52wk high strategy results reported in Table 2, the arbitrage profits in Table 7 are at least $0.5 \%$ per month larger than the corresponding entries in Table 2.

Of particular interest, the average returns for the short portfolios (LSV) of the modified strategy in Table 7 are consistently less than the corresponding entries for the short portfolios $(L)$ reported in Table 2 . This suggests that the volatility explanation for the failure of the 52wk high strategy is at least partially successful. Note, however, that the arbitrage results for the modified 52wk high strategy in Table 8 are each less than the corresponding pure momentum results reported in Table 3 . This means that the modifications to the $52 \mathrm{wk}$ high strategy are not sufficient to make it as profitable as pure momentum.

$<$ Table 7 about here>

Risk-adjusted results for the modified strategy are presented in Table 8. The results are dramatic. Whereas the short portfolio $(L)$ in Table 6 produced significant alphas exceeding 14\%, the short portfolio (LSV) in Table 8 has insignificant alphas of $4 \%$ or $4.9 \%$. Volatility considerations have succeeded in eliminating the abnormal returns of the short portfolio in the standard 52wk high strategy. On the other hand, while the profitability of the 
arbitrage portfolio $H B v-L S v$ produces positive alphas of about $6 \%$, they are not significant. ${ }^{8}$ In summary, while our modifications based on volatility have been enough to explain the abnormally high returns of 52wk high's short portfolio, they have not been enough to make the modified 52wk high strategy as profitable as the pure momentum strategy.

$<$ Table 8 about here $>$

\section{Discussion and Conclusion}

This study has focused on whether the 52wk high strategy can be profitably applied to emerging market indices. Our study answers this question in the negative. The $52 w \mathrm{k}$ strategy makes losses when applied to emerging markets indices, meaning that price levels are not useful in explaining future returns in the way predicted by the $52 \mathrm{wk}$ high effect. In contrast, momentum strategies applied to emerging markets indices are profitable.

In the case of individual stocks, George and Hwang (2004) adopt an 'adjustment and anchoring bias' explanation for the $52 \mathrm{wk}$ high effect in which traders use the $52 \mathrm{wk}$ high price as an anchor when adjusting stock values as new information arrives. As good (bad) news pushes the price of a stock closer to (farther from) a new high, investors are initially reluctant to bid the price up (down) sufficiently. Eventually the information pushes through causing the prices to move up (down) appropriately. This short term underreaction causes a continuation in prices which the market corrects without causing long-term reversals.

The evidence presented in this paper suggests that this theory cannot be successfully applied to emerging market indices. It appears that the 52wk high price level of an emerging market index does not provide the same anchor to international index investors as George and Hwang (2004) claim a stock's 52wk high does to share investors.

The problem with the 52wk high strategy comes from the strategy's short portfolio which is composed of those emerging markets indices with prices far from their 52wk highs.

\footnotetext{
${ }^{8}$ Similarly, although the corresponding alphas for the pure momentum strategy exceed $10 \%$, they also are not significant (results available on request).
} 
Instead of having low future returns, such indices tend to have abnormally high future returns. We put forward and test one possible explanation for this empirical observation. The extremely poor returns which result in indices being included in the short portfolio may simply be indicative of exceptional volatility. Since high volatility is positively associated with high average returns, then the short portfolio's abnormally high future returns could be due to it unintentionally containing many of the more highly volatile of the emerging market indices.

Our tests support this explanation. When the short portfolio is modified to include only indices with low recent volatility, the resulting portfolio no longer has abnormally high future returns. Yet even this modified $52 w k$ high strategy is less profitable than the corresponding momentum strategy. Overall, our study suggests that the 52wk high effect is not as pervasive an effect as the widely observed momentum effect. 


\section{References}

Balvers, R. J., \& Wu, Y. (2006). Momentum and Mean Reversion Across National Equity Markets. Journal of Empirical Finance, 13, 24-48.

Barberis, N., Shleifer, A., \& Vishny, R. (1998). A Model of Investor Sentiment. Journal of Financial Economics, 49, 307-343.

Bhojraj, S., \& Swaminathan, B. (2006). Macromomentum:Evidence of Predictability in International Equity Markets. Journal of Business, 79(1), 429-451.

Chan, K., Hameed, A., \& Tong, W. (2000). Profitability of Momentum Strategies in the International Equity Markets. Journal of Financial and Quantitative Analysis, 35(2), 153-173.

Chan, L. K. C., Jegadeesh, N., \& Lakonishok, J. (1999). The Profitability of Momentum Strategies. Financial Analysts Journal, 55(6), 80-90.

Daniel, K. D., Hirshleifer, D., \& Subrahmanyam, A. (1998). Investor Psychology and Security Market Under- and Overreactions. Journal of Finance, 53, 1839-1887.

Daniel, K. D., \& Titman, S. (1999). Market Efficiency in an Irrational World. Financial Analysts Journal, 55(6), 28-40.

Du, D. (2008). The 52-week high and momentum investing in international stock indices. The Quarterly Review of Economics and Finance, 48, 61-77.

Fama, E. F., \& French, K. R. (1996). Multifactor Explanation of Asset Pricing Anomalies. Journal of Finance, 51(1), 55-84.

George, T. J., \& Hwang, C.-Y. (2004). The 52-Week High and Momentum Investing. Journal of Finance, 59(5), 2145-2176.

Griffin, J. M., Ji, X., \& Martin, J. S. (2003). Momentum Investing and Business Cycle Risk: Evidence from Pole to Pole. Journal of Finance, 58(6), 2515-2548.

Grundy, B. D., \& Martin, S. J. (2001). Understanding the Nature of the Risks and the Source of the Rewards to Momentum Investing. Review of Financial Studies, 14(1), 29-78. 
Hong, H., \& Stein, J. C. (1999). A Unified Theory of Underrreaction, Momentum Trading and Overreaction in Asset Markets. Journal of Finance, 54, 2143-2184.

Jegadeesh, N., \& Titman, S. (1993). Returns to Buying Winners and Selling Losers:

Implications for Stock Market Efficiency. Journal of Finance, 48(1), 65-91.

Lee, C. M. C., \& Swaminathan, B. (2000). Price Momentum and Trading Volume. Journal of Finance, 55(5), 2017-2069.

Lewellen, J. (2002). Momentum and Autocorrelation in Stock Returns. Review of Financial Studies(15), 533-563.

Marshall, B. R., \& Cahan, R. M. (2005). Is the 52-week High Momentum Strategy Profitable Outside the US? Applied Financial Economics, 15(18), 1259-1267.

Moskowitz, T. J., \& Grinblatt, M. (1999). Do Industries Explain Momentum? Journal of Finance, 54(4), 1249-1290.

Rouwenhorst, K. G. (1998). International Momentum Strategies. Journal of Finance, 53(1), 267-284. 